\title{
Correction to: Lipid accumulation in prokaryotic microorganisms from arid habitats
}

\author{
Philippa Hauschild ${ }^{1}$ (D) - Annika Röttig ${ }^{1} \cdot$ Mohamed H. Madkour ${ }^{2} \cdot$ Ahmed M. Al-Ansari $^{2} \cdot$ Naief H. Almakishah $^{2}$ • \\ Alexander Steinbüchel ${ }^{1,2}$
}

Published online: 3 February 2020

(C) Springer-Verlag GmbH Germany, part of Springer Nature 2020

\section{Correction to: Appl Microbiol Biotechnol (2017) 101:2203-2216} https://doi.org/10.1007/s00253-017-8149-0

There is an error in the Original Publication of this paper for "Acknowledgements" section was missing.

Acknowledgements This project was funded by the Deanship of Scientific Research (DSR) at King Abdulaziz University, Jeddah, under grant no. (2-155-34-HiCi). The authors, therefore, acknowledge with thanks DSR for technical and financial support.

Publisher's note Springer Nature remains neutral with regard to jurisdictional claims in published maps and institutional affiliations.

The online version of the original article can be found at https://doi.org/ $10.1007 / \mathrm{s} 00253-017-8149-0$

Alexander Steinbüchel

steinbu@uni-muenster.de

1 Institut für Molekulare Mikrobiologie und Biotechnologie,

Westfälische Wilhelms-Universität Münster, Corrensstraße 3,

D-48149 Münster, Germany

2 Environmental Sciences Department, Faculty of Meteorology,

Environment and Arid Land Agriculture, King Abdulaziz University, Jeddah 21589, Saudi Arabia 